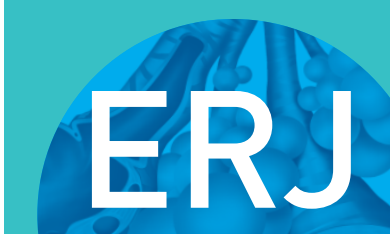

open research

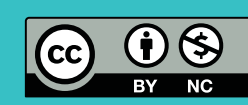

\section{Importance of computed tomography in defining segmental disease in chronic thromboembolic pulmonary hypertension}

\author{
Micheal C. Mclnnis (1) ${ }^{1}$, David Wang ${ }^{1}$, Laura Donahoe ${ }^{2}$, John Granton ${ }^{3}$, \\ John Thenganatt ${ }^{3}$, Kongteng Tan ${ }^{1}$, John Kavanagh ${ }^{1}$ and Marc de Perrot ${ }^{2}$
}

Affiliations: ${ }^{1}$ Dept of Medical Imaging, University of Toronto, Toronto, ON, Canada. ${ }^{2}$ Division of Thoracic Surgery, Dept of Surgery, University of Toronto, Toronto, ON, Canada. ${ }^{3}$ Division of Respirology, Dept of Medicine, University of Toronto, Toronto, ON, Canada.

Correspondence: Micheal C. Mclnnis, Dept of Medical Imaging, Toronto General Hospital, 1 PMB-273, 585 University Avenue, Toronto, ON M5G 2N2, Canada. E-mail: Micheal.mcinnisQunn.ca

\section{ABSTRACT}

Background: Radiological assessment of patients with chronic thromboembolic pulmonary hypertension (CTEPH) is critical to decide whether patients should be treated with pulmonary endarterectomy (PEA). Although computed tomography pulmonary angiography (CTPA) is increasingly used for decision making in CTEPH, the value of CTPA to predict surgical findings and outcome has never been explored.

Methods: We retrospectively reviewed 100 consecutive patients with high-quality CTPA undergoing PEA for CTEPH between May 2015 and December 2017. The most proximal level of disease in the pulmonary artery on CTPA was classified by two blinded radiologists as level 1 (main pulmonary artery), 2a (lobar pulmonary artery), $2 \mathrm{~b}$ (origin of basal segmental pulmonary artery), 3 (segmental pulmonary artery) or 4 (predominantly subsegmental pulmonary artery).

Results: CTPA demonstrated level 1 in 20\%, level 2a in 43\%, level 2b in 11\%, level 3 in $23 \%$ and level 4 in $3 \%$. A majority of males presented with level 1 (55\%) and level 2 (57\%), and a majority of females (83\%) with level $3(\mathrm{p}=0.01)$. Levels 3 and 4 were associated with longer duration of circulatory arrest $(\mathrm{p}=0.03)$ and higher frequency of Jamieson type III disease at surgery $(\mathrm{p}<0.0001)$. Requirement for targeted pulmonary hypertension therapy after PEA was $28 \%$ at 3 years in level $2 \mathrm{~b} / 3 / 4$ compared with $6 \%$ in level $2 \mathrm{a}$ and $13 \%$ in level $1(\mathrm{p}=0.002)$. Level $2 \mathrm{~b} / 3 / 4$ was an independent predictor for targeted pulmonary hypertension therapy after PEA (hazard ratio 4.23 , 95\% CI 1.24-14.39; $\mathrm{p}=0.02$ ).

Conclusions: High-quality CTPA provides accurate evaluation of CTEPH patients. The level of disease on CTPA can help guide peri-operative planning and post-operative monitoring.

@ERSpublications

High-quality CTPA provides accurate evaluation of CTEPH patients. The level of disease on CTPA can predict surgical difficulty and the need for targeted PH therapy after PEA. https://bit.ly/317ZBSu

Cite this article as: McInnis MC, Wang D, Donahoe L, et al. Importance of computed tomography in defining segmental disease in chronic thromboembolic pulmonary hypertension. ERJ Open Res 2020; 6: 00461-2020 [https://doi.org/10.1183/23120541.00461-2020].

Received: 2 July 2020 | Accepted after revision: 9 Oct 2020

Copyright $\odot$ ERS 2020. This article is open access and distributed under the terms of the Creative Commons Attribution Non-Commercial Licence 4.0. 


\section{Introduction}

Chronic thromboembolic pulmonary hypertension (CTEPH) is an underdiagnosed disease that occurs in $\sim 4 \%$ of subjects at 2 years following acute pulmonary embolism [1]. Subjects present with insidious symptoms and there is frequently a delay in diagnosis [2]. A diagnosis of CTEPH is associated with significant morbidity and mortality [3]. The prognosis for patients with CTEPH is poor in the absence of treatment. Targeted medical therapy can be very effective, but is not curative [4]. Pulmonary endarterectomy (PEA) is the only curative therapy for CTEPH and survival as well as quality of life in operated patients is excellent compared with conservative management $[3,5]$. Therefore, identification of surgical candidates is important in maximising patient outcomes [6]. More recently, balloon pulmonary angioplasty (BPA) has also become an integral part of the care of patients with CTEPH who are not candidates for PEA, further emphasising the importance of high-quality imaging for diagnosis and therapeutic decision making in CTEPH.

An international prospective registry, which collected patients between 2007 and 2009, observed that subjects undergoing PEA had excellent post-operative outcomes, but that a large proportion of patients with segmental disease were excluded from surgery [3]. However, with improving imaging and surgical techniques, patients with segmental disease have been increasingly treated with PEA in expert centres with excellent results $[7,8]$. Hence, optimal radiological evaluation of CTEPH patients is critical to determine surgical candidacy, particularly at a segmental level.

The computed tomography (CT) imaging features of CTEPH have been previously described [9]. However, the value of CT pulmonary angiography (CTPA) in predicting surgical findings and post-operative outcome has not been analysed systematically. This point is of importance considering that patients with segmental disease are increasingly treated surgically and that CTPA is progressively replacing pulmonary angiography to assess surgical candidacy. Hence, determining the reliability of CT is important because of the central role it plays in the selection of subjects eligible for surgery.

\section{Materials and methods}

\section{Subject selection}

The study included all adults with a clinical diagnosis of CTEPH who underwent PEA at Toronto General Hospital (Toronto, ON, Canada) between April 30, 2015 and December 31, 2017. Confirmation of diagnosis and eligibility for surgery was assessed in multidisciplinary rounds including a thoracic radiologist, thoracic surgeons and respirologists with expertise in pulmonary hypertension. The Institutional Review Board approved this retrospective study.

\section{CT imaging}

Our standard protocol for evaluation of CTEPH is performed during suspended respiration. We inject $70 \mathrm{~cm}^{3}$ of iodinated contrast (Ultravist 370; Bayer Healthcare, Berlin, Germany) at $5 \mathrm{~cm}^{3} \cdot \mathrm{s}^{-1}$ via an $18 \mathrm{G}$ antecubital intravenous catheter. The acquisition is triggered at $200-250 \mathrm{HU}$ using bolus tracking software with a region of interest placed over the main pulmonary artery. A standard voltage is set at $120 \mathrm{kV}$ in most patients and automated exposure control determines the current. CT images are reconstructed in $0.5-\mathrm{mm}$ thick axial slices using a mediastinal kernel. Coronal and sagittal reformats are performed with $1-\mathrm{mm}$ thick slices using a mediastinal kernel. Coronal maximum intensity projections with 8 - $\mathrm{mm}$ thick slices are performed.

Not all subjects had a CTPA performed at our institution because many were referred from other institutions. One reader (M.M.) reviewed all internal and external CTPA exams for technical adequacy. At a minimum, a CTPA included in this study had to demonstrate adequate opacification of the pulmonary arteries ( $\geqslant 300 \mathrm{HU}$ measured over the main pulmonary artery). The study must have included axial slices in a mediastinal reconstruction kernel with $\leqslant 1.5 \mathrm{~mm}$ thickness and multiplanar reformats. Studies with significant artefacts (e.g. respiratory motion, contrast mixing artefacts or artefacts from hardware) limiting diagnostic quality were excluded.

\section{Image analysis}

The CTPA closest to the date of surgery was chosen for review. A fellowship trained thoracic radiologist (M.M.) and a diagnostic radiology trainee (D.W.) blinded to the patient's outcome independently reviewed each case individually for the presence of chronic pulmonary embolism. The two readers then reviewed cases with disagreement to come to a consensus read used for analysis. CT images were viewed on a picture archiving and communication system (Coral, Toronto, ON, Canada). Images were reviewed on the thinnest available axial slice. Multiplanar reformats were available for review when needed. The following findings were considered indicative of chronic pulmonary embolism: occlusions or pouch defects, slits and webs, or eccentric thrombus (with or without calcification). Occlusions describe the complete occlusion of a pulmonary artery at its origin, usually associated with a distal diminutive vessel. Pouch defects are 
occlusions but with a small contrast-opacified pouch at the vessel origin. A slit is a single fibrous band extending across the lumen of a vessel, often at the vessel origin. A web is a network of slits. Eccentric thrombus corresponds to an eccentric filling defect along the margin of a vessel, occasionally calcified [9].

The radiological level of disease was assigned based on the most proximal disease present. Level 1 disease was defined as any disease in the main, left or right main pulmonary artery. Level 2 disease was defined as any disease in the interlobar or lobar pulmonary vessels down to the origin of the segmental vessels. Level 2 was then subdivided into levels $2 \mathrm{a}$ and $2 \mathrm{~b}$ for increased precision. Level $2 \mathrm{~b}$ disease was defined as disease in the basal trunk distal to the superior segmental pulmonary artery, most often around the origin of the basal segmental vessels. Level 2a was disease in any lobar vessel, including the superior segment in the lower lobes. We did this subdivision considering that the take-off of the segmental arteries in the lower lobes is an important landmark in assessing surgical disease, that this is a common location of chronic pulmonary embolism, and that the basal trunk is anatomically distinct from a lobar vessel and a segmental vessel. Level 3 disease was defined as disease starting in the segmental pulmonary arteries within $1 \mathrm{~cm}$ of its origin, including disease in the basal lower lobes segmental arteries that does not extend proximally into the basal trunk. Level 4 disease was defined as disease starting more than $1 \mathrm{~cm}$ from the origin of the segmental vessel and predominantly located in the subsegmental vessels. In cases where one pulmonary segment was supplied by two independent vessels, these vessels were each classified as subsegmental.

\section{Surgical findings}

PEA was performed using the standardised technique previously described with periods of deep hypothermic circulatory arrest at $20^{\circ} \mathrm{C}$ [7]. The surgical specimens were routinely classified into types I-IV based on the Jamieson classification at the time of surgery [10]. Briefly, type I was defined by the presence of fresh or subacute thromboembolic material in the main and lobar pulmonary artery. Type II was defined by the presence of organised thromboembolic and fibrotic material in the lobar arteries without any fresh clot. Type III was defined by the presence of organised thromboembolic and fibrotic material in the segmental and subsegmental branches only. Type IV was defined by the absence of organised thromboembolic material.

\section{Follow-up after PEA}

All patients followed-up in our institution were included in the long-term analysis. Follow-up was performed at 3, 6 and 12 months, and then on a yearly basis. Patients were started on targeted pulmonary hypertension therapy in the presence of pre-capillary pulmonary hypertension on right heart catheterisation combined with persistent New York Heart Association functional class III or IV after PEA or worsening of at least one functional class compared with the best class achieved after PEA.

\section{Statistical analysis}

A two-tailed p-value of $<0.05$ was considered statistically significant. Continuous variables were described using median (range) or mean with standard deviation, and categorical variables were described using number (percentage). Comparisons between groups were made by the independent samples t-test or one-way ANOVA for continuous variables and Fisher's exact test or Chi-squared test for categorical variables. Cumulative survival was calculated from the date of surgery. Cumulative proportion of patients requiring targeted pulmonary hypertension therapy after PEA was calculated using life table analysis. Groups were compared using the log-rank test. Multivariate Cox regression analysis was used to determine pre-operative factors associated with the need for targeted pulmonary hypertension therapy during follow-up after PEA.

\section{Results}

\section{Subject population}

We identified 113 consecutive subjects who underwent PEA between April 30, 2015 and December 31, 2017, all of whom had a pre-operative CTPA. 13 subjects were excluded due to mixing artefacts in the pulmonary arteries $(\mathrm{n}=4)$, axial CTPA slice thickness $>1.5 \mathrm{~mm}(\mathrm{n}=3)$, inadequate pulmonary artery opacification $<300 \mathrm{HU}(\mathrm{n}=3)$, significant respiratory motion $(\mathrm{n}=2)$ and respiratory motion with mixing artefacts $(\mathrm{n}=1)$. The remaining 100 subjects all had complete data and were included in the final analysis. There was no significant difference in terms of clinical characteristics between included and excluded patients with the exception of higher brain natriuretic peptide (BNP), right atrial pressures and diastolic pulmonary arterial pressures in the excluded group (table 1).

Among the 100 patients included in the study, the median (range) time between CTPA and PEA was 18 (1-615) days (96\% within 6 months). In 91\% of subjects, the CTPA closest to the date of surgery was performed at our institution. Axial slice thickness of $<1 \mathrm{~mm}$ in a mediastinal kernel was available for 


\begin{tabular}{|c|c|c|c|}
\hline & Included patients & Excluded patients & p-value \\
\hline Patients & 100 & 13 & \\
\hline Sex & & & 0.6 \\
\hline Male & 48 & 5 & \\
\hline Female & 52 & 8 & \\
\hline Age years & $58 \pm 15$ & $61 \pm 13$ & 0.5 \\
\hline Functional class & $2.8 \pm 0.7$ & $3.2 \pm 0.8$ & 0.06 \\
\hline 6MWD m & $380 \pm 153$ & $352 \pm 179$ & 0.5 \\
\hline BNP $\mathrm{pg} \cdot \mathrm{mL}^{-1}$ & $253 \pm 378$ & $623 \pm 835$ & 0.006 \\
\hline Mean PAP mmHg & $43 \pm 13$ & $48 \pm 12$ & 0.2 \\
\hline Systolic PAP mmHg & $71 \pm 21$ & $77 \pm 19$ & 0.3 \\
\hline Diastolic PAP mmHg & $26 \pm 9$ & $31 \pm 4$ & 0.05 \\
\hline RAP mmHg & $10 \pm 5$ & $13 \pm 4$ & 0.04 \\
\hline PCWP mmHg & $11 \pm 4$ & $11 \pm 6$ & 0.1 \\
\hline Cardiac index L-min $\cdot \mathrm{m}^{-2}$ & $2.1 \pm 0.5$ & $2.2 \pm 0.8$ & 0.5 \\
\hline PVR dyn $\cdot s \cdot \mathrm{cm}^{-5}$ & $714 \pm 422$ & $832 \pm 497$ & 0.4 \\
\hline Pre-operative PH therapy & 23 & 3 & 1.0 \\
\hline Type of disease ( Jamieson) & & & 0.4 \\
\hline Type I & 26 & 5 & \\
\hline Type II & 30 & 3 & \\
\hline Type III & 44 & 5 & \\
\hline Hospital mortality after PEA & 1 & 0 & 1.0 \\
\hline \multicolumn{4}{|c|}{$\begin{array}{l}\text { Data are presented as } n \text { or mean } \pm S D \text {, unless otherwise stated. } 6 \mathrm{MWD} \text { : } 6 \text {-min walk distance; BNP: brain } \\
\text { natriuretic peptide; PAP: pulmonary arterial pressure; RAP: right atrial pressure; PCWP: pulmonary } \\
\text { capillary wedge pressure; PVR: pulmonary vascular resistance; } \mathrm{PH} \text { : pulmonary hypertension; PEA: } \\
\text { pulmonary endarterectomy. }\end{array}$} \\
\hline
\end{tabular}

review in $92 \%$ of all subjects. A slice thickness of $1 \mathrm{~mm}$ was available in $7 \%$ and $1 \%$ had a slice thickness of $1.3 \mathrm{~mm}$.

\section{CTPA and clinical characteristics}

Of the 100 patients with adequate pre-operative CTPA, 20 were classified with level 1, 54 with level 2, 23 with level 3 and three with level 4 . Overall, 74\% of the CTPA levels were agreed upon by both radiologists (weighted $\kappa=0.64$ ), with $70 \%$ of disagreements related to a failure to recognise more proximal disease. Patient characteristics differed based on the level of disease (table 2). The proportion of females was significantly higher in level $3(83 \%)$, while the proportion of males was higher in level $2(57 \%)$ and level 1 (55\%). Patients with level 1 had lower 6-min walk distance (6MWD), and higher BNP and pulmonary vascular resistance (PVR) compared with patients with level 2 and level 3. Patients with level 4 walked further on their 6MWD, and had the lowest BNP and PVR values. Patients with level 3 had significantly lower 6MWD compared with patients with level $2(\mathrm{p}=0.01)$, but there was no significant difference in BNP and PVR between these two groups. Patients with level 3 and level 4 were more frequently treated with targeted pulmonary hypertension therapy pre-operatively compared with patients with level 1 and level 2.

\section{Surgical findings and outcome}

At the time of surgery, 26 patients were classified with Jamieson type I disease, 30 with Jamieson type II disease and 44 with Jamieson type III disease. The mean \pm SD time of circulatory arrest progressively increased with the level of disease determined on CTPA from $41 \pm 10 \mathrm{~min}$ for level 1 to $47 \pm 13$ min for level 2, $51 \pm 12 \mathrm{~min}$ for level 3 and $57 \pm 17 \mathrm{~min}$ for level 4 (table 3 ). Significant improvement in total pulmonary resistance between the pre-operative value and the first post-operative day after PEA was observed for each level of disease besides level 4 (figure 1). The duration of intubation, length of intensive care unit stay and length of hospital stay was similar for each level of disease (table 3).

\section{Radiological-pathological correlation}

There was a significant association between the level of disease on CTPA and the Jamieson classification found at surgery (figure 2). $70 \%$ of patients with level 1 on CTPA had Jamieson type I disease at surgery, $43 \%$ of those with level 2 had Jamieson type II disease, $87 \%$ of those with level 3 had Jamieson type III disease and all three of those with level 4 had Jamieson type III disease at surgery $(\mathrm{p}<0.0001)$. Level 2 on 


\begin{tabular}{|c|c|c|c|c|c|}
\hline & Level 1 & Level 2 & Level 3 & Level 4 & p-value \\
\hline Patients & 20 & 54 & 23 & 3 & \\
\hline Female & $9(45)$ & $23(43)$ & $19(83)$ & 1 (33) & 0.01 \\
\hline Age years & $60 \pm 16$ & $57 \pm 13$ & $59 \pm 17$ & $42 \pm 16$ & 0.2 \\
\hline Functional class & $2.8 \pm 0.8$ & $2.7 \pm 0.7$ & $3.0 \pm 0.5$ & $3.0 \pm 0.0$ & 0.3 \\
\hline 6MWD m & $319 \pm 147$ & $420 \pm 161$ & $327 \pm 114$ & $475 \pm 99$ & 0.01 \\
\hline BNP $\mathrm{pg} \cdot \mathrm{mL}^{-1}$ & $460 \pm 508$ & $190 \pm 299$ & $243 \pm 383$ & $49 \pm 66$ & 0.04 \\
\hline Mean PAP mmHg & $48 \pm 15$ & $41 \pm 12$ & $45 \pm 12$ & $33 \pm 13$ & 0.08 \\
\hline Systolic PAP mmHg & $79 \pm 21$ & $68 \pm 20$ & $74 \pm 23$ & $55 \pm 22$ & 0.1 \\
\hline Diastolic PAP $\mathrm{mmHg}$ & $30 \pm 12$ & $25 \pm 8$ & $26 \pm 8$ & $20 \pm 9$ & 0.1 \\
\hline RAP $\mathrm{mmHg}$ & $11 \pm 6$ & $10 \pm 4$ & $11 \pm 5$ & $5 \pm 2$ & 0.2 \\
\hline PCWP mmHg & $12 \pm 3$ & $12 \pm 4$ & $10 \pm 4$ & $7 \pm 2$ & 0.03 \\
\hline Cardiac index L.min $\cdot \mathrm{m}^{-2}$ & $1.8 \pm 0.5$ & $2.1 \pm 0.5$ & $2.4 \pm 0.7$ & $2.5 \pm 0.3$ & 0.001 \\
\hline PVR dyn $\cdot \mathrm{s} \cdot \mathrm{cm}^{-5}$ & $1029 \pm 556$ & $611 \pm 335$ & $731 \pm 387$ & $458 \pm 241$ & 0.001 \\
\hline Pre-operative echo RV size & & & & & 0.9 \\
\hline Normal & 2 & 11 & 5 & 1 & \\
\hline Mild & 2 & 10 & 4 & 1 & \\
\hline Moderate & 5 & 14 & 5 & 0 & \\
\hline Severe & 9 & 15 & 8 & 1 & \\
\hline Pre-operative echo RV function & & & & & 0.7 \\
\hline Normal & 4 & 18 & 5 & 1 & \\
\hline Mild & 3 & 9 & 2 & 0 & \\
\hline Moderate & 6 & 15 & 11 & 2 & \\
\hline Severe & 4 & 7 & 4 & 0 & \\
\hline Pre-operative PH therapy & $3(15)$ & 9 (17) & 9 (39) & $2(67)$ & 0.04 \\
\hline
\end{tabular}

Data are presented as $\mathrm{n}, \mathrm{n}(\%)$ or mean $\pm \mathrm{SD}$, unless otherwise stated. 6MWD: 6-min walk distance; BNP: brain natriuretic peptide; PAP: pulmonary arterial pressure; RAP: right atrial pressure; PCWP: pulmonary capillary wedge pressure; $\mathrm{Cl}$ : cardiac index; PVR: pulmonary vascular resistance; echo: echocardiogram; $\mathrm{RV}$ : right ventricular; $\mathrm{PH}$ : pulmonary hypertension.

CTPA was the least predictive of the type of disease found at surgery, with $20 \%$ Jamieson type I disease, $43 \%$ Jamieson type II disease and 37\% Jamieson type III. Dividing level 2 on CTPA into level 2a and $2 \mathrm{~b}$ provided better correlation with the surgical finding. Jamieson type II disease was found in $46 \%$ of patients with level $2 \mathrm{a}$ on CTPA and $27 \%$ of those with level $2 \mathrm{~b}$, while Jamieson type III disease was identified in

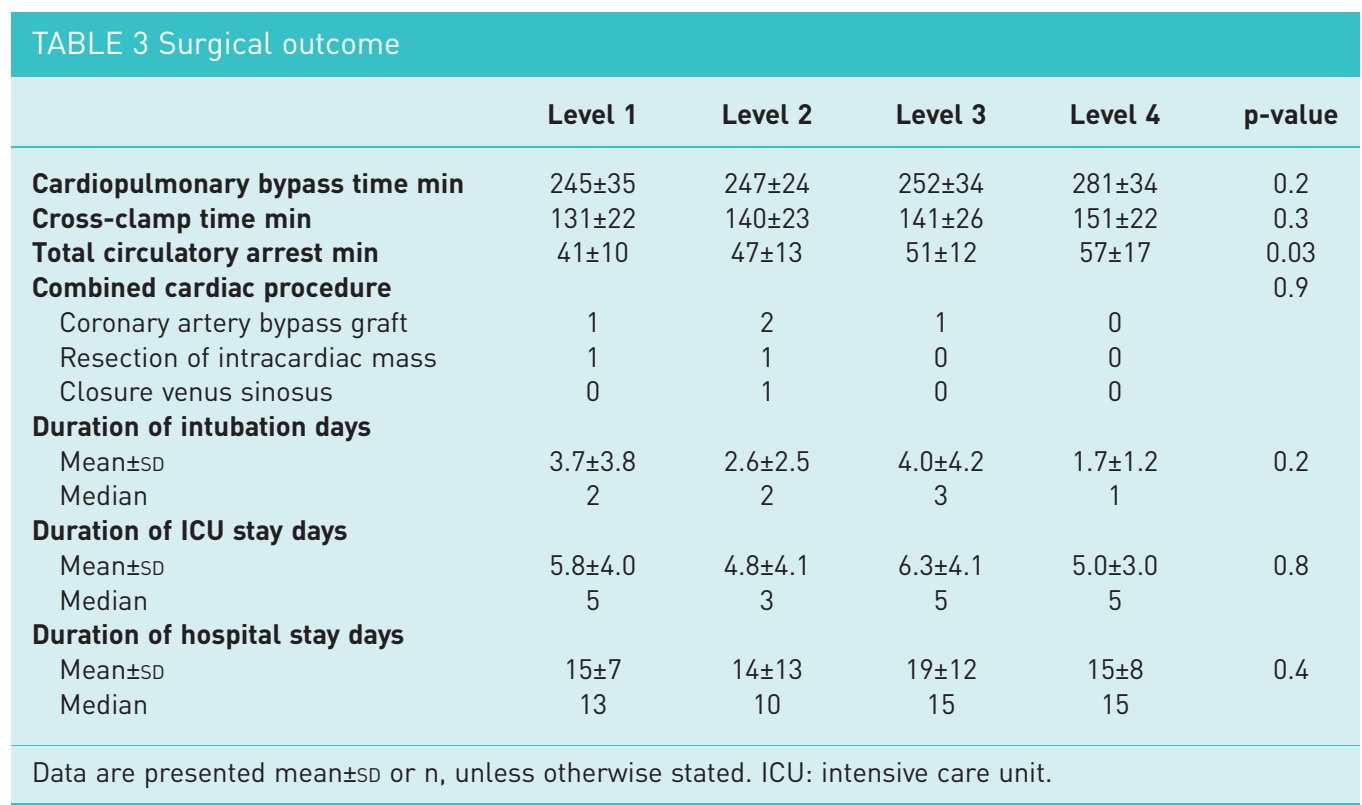




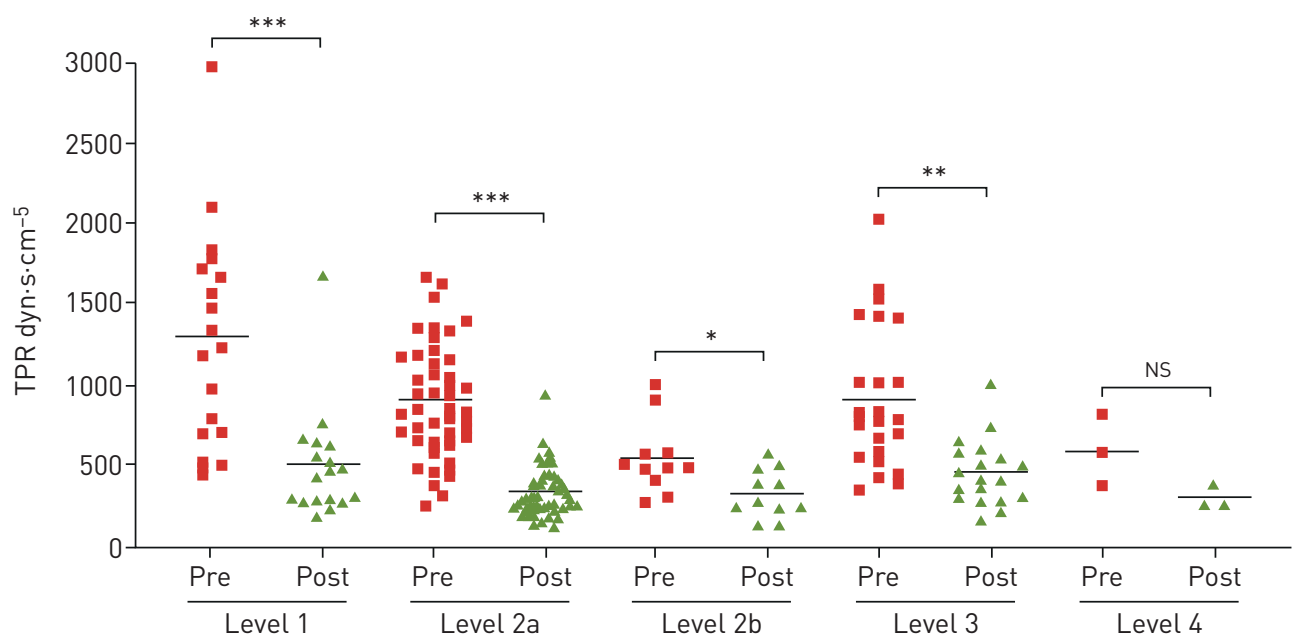

FIGURE 1 Improvement in total pulmonary resistance (TPR) according to the level of disease on computed tomography pulmonary angiography. Pre: pre-operative TPR calculated on the pre-operative right heart catheterisation; post: post-operative TPR calculated on the first post-operative day after pulmonary endarterectomy; NS: nonsignificant. NS: $p=0.1 ;{ }^{*}: p=0.02 ;{ }^{* *}: p=0.0002 ;{ }^{* * *}: p<0.0001$.

$28 \%$ of patients with level $2 \mathrm{a}$ and $73 \%$ of those with level $2 \mathrm{~b}$. Overall, CTPA was most accurate in predicting segmental disease, with the likelihood of finding Jamieson type III disease at surgery reaching $73 \%$ for level $2 \mathrm{~b}, 87 \%$ for level 3 and $100 \%$ for level 4

\section{Long-term outcome}

After a median (range) follow-up of $34(1-54)$ months, six patients died for an overall survival of $93 \%$ at 3 years. Survival was similar based on the level of disease on CTPA (figure 3). A total of 14 patients were started on targeted pulmonary hypertension therapy for persistent or worsening symptoms and residual pre-capillary pulmonary hypertension after PEA. The use of targeted pulmonary hypertension therapy was significantly higher in patients with segmental disease on CTPA, reaching $28 \%$ at 3 years in level $2 \mathrm{~b} / 3 / 4$ compared with $6 \%$ in level $2 \mathrm{a}$ and $13 \%$ in patients with level 1 (figure 3 ). In multivariate analysis, the level of disease on CTPA was an independent predictor for targeted pulmonary hypertension therapy after PEA (table 4).

\section{Discussion}

This study demonstrates the importance of CTPA in the evaluation of CTEPH patients for PEA. We also showed that excellent quality CTPA is particularly beneficial in patients with segmental disease. Evidence

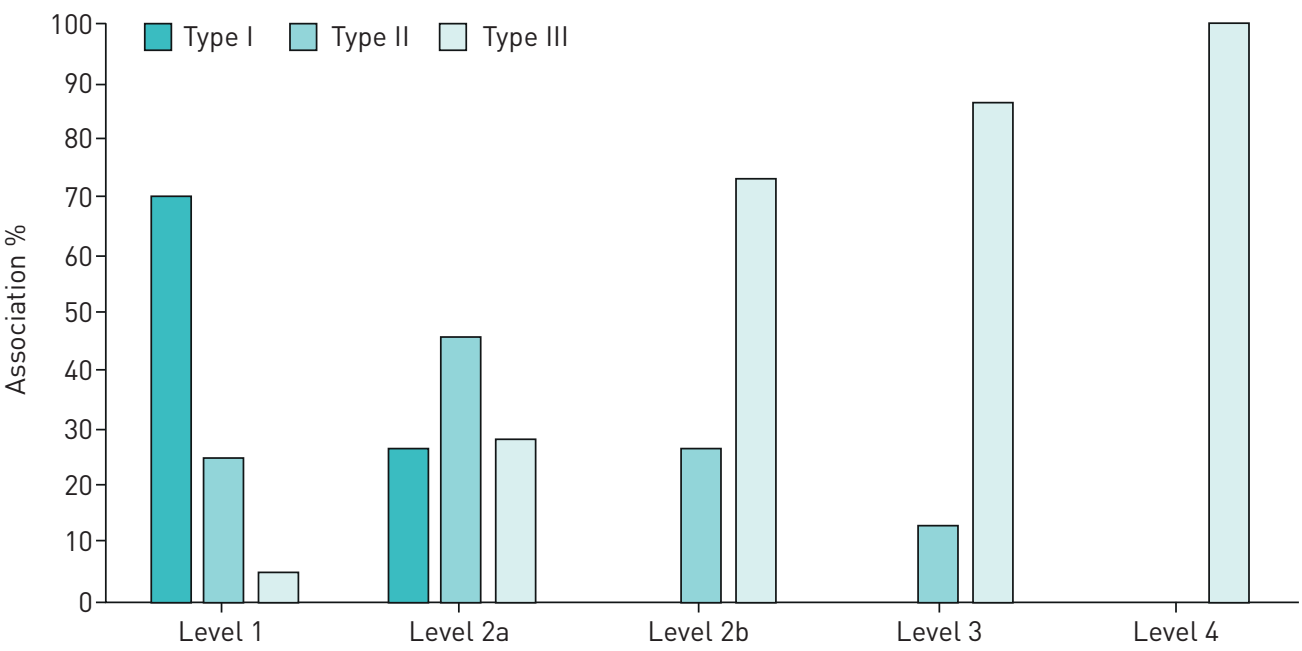

FIGURE 2 Distribution of the type of surgical disease based on the Jamieson type classification compared with the level of disease detected by computed tomography pulmonary angiography. 
a)

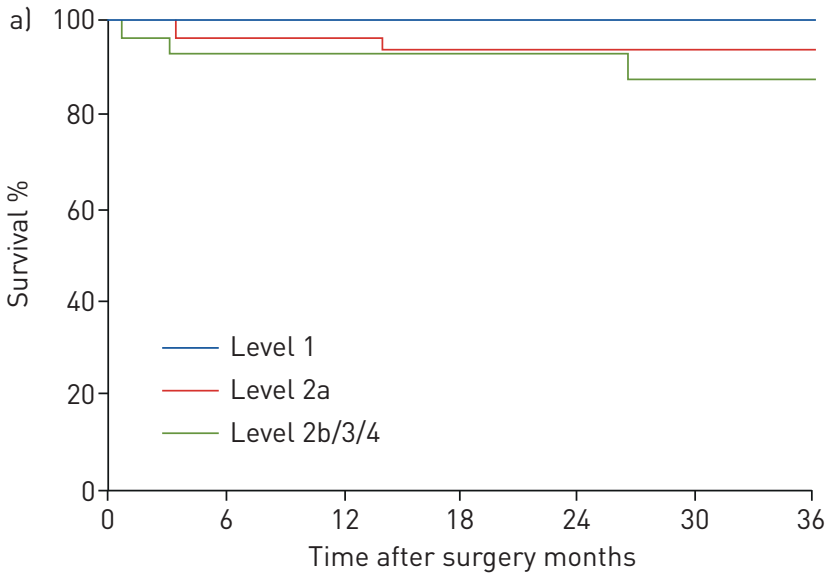

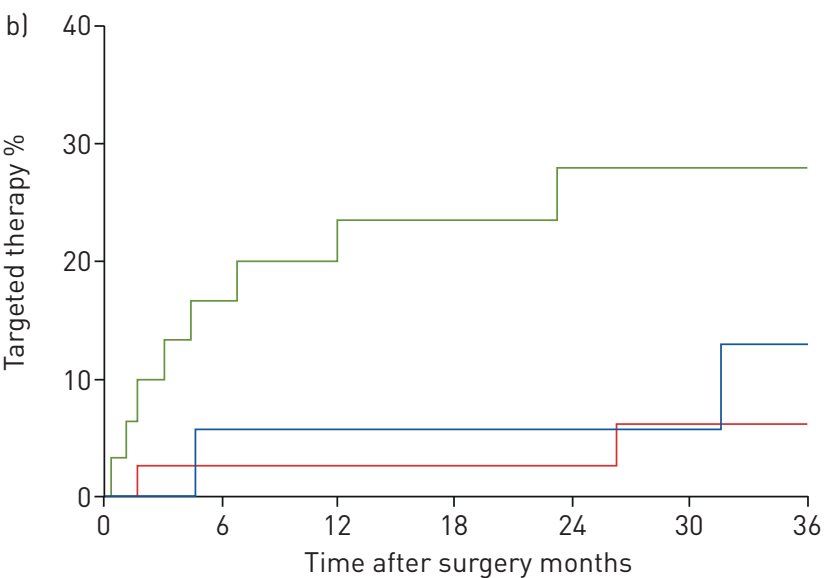

FIGURE 3 a) Survival after pulmonary endarterectomy (PEA) according to the level of disease on computed tomography pulmonary angiography $(p=0.3)$. b) Percentage of patients requiring targeted pulmonary hypertension therapy after PEA ( $p=0.02)$.

of chronic thromboembolic disease at the ostium of the basal segmental branches of the pulmonary artery on CTPA (level 2b) was associated with Jamieson type III disease at surgery in $73 \%$ of the patients and evidence of chronic thromboembolic disease within the segmental artery only was associated with the presence of Jamieson type III disease at surgery in $87 \%$ of the patients. Although very few patients with chronic thromboembolic disease located in the distal segmental or subsegmental arteries (level 4) were included, all these patients had Jamieson type III disease at surgery. The importance of CTPA in the evaluation of patients with CTEPH is also demonstrated by the impact of the level of disease on the duration of circulatory arrest and the need for targeted pulmonary hypertension therapy in the long term after surgery.

Hence, accurate pre-operative classification of the level of disease on high-quality CTPA is important and reliable, particularly in patients with segmental disease. PEA for segmental disease can be technically more challenging and correct classification of disease allows optimal pre-operative planning. Pre-operative imaging is critical to obtain the correct diagnosis and to predict surgical findings. This is particularly true at a segmental level where a misdiagnosis can lead to very difficult post-operative management due to the presence of residual pulmonary hypertension in the context of right ventricular dysfunction. As shown in this study and others, the early and long-term outcomes of patients undergoing PEA can be excellent even in patients with segmental disease $[8,11]$. Despite this, there are physiological differences in segmental disease and these patients are more likely to require targeted pulmonary hypertension therapy in the long term compared with patients with more proximal disease. Hence, a correct classification of segmental disease on CTPA remains important even in the long term, emphasising the value of classifying these patients adequately upfront [12].

CTPA has an important role in the evaluation of CTEPH in the setting of acute and chronic pulmonary emboli. Although a ventilation/perfusion scan remains the recommended imaging modality for screening for CTEPH, CTPA is routinely used to confirm the presence of chronic pulmonary emboli in patients with an abnormal ventilation/perfusion scan [2]. In addition, although its role is underreported, CTPA may also identify patients with CTEPH at the time of acute pulmonary embolism where CTEPH was unsuspected $[13,14]$. Compared with conventional angiography, CTPA is noninvasive, faster to perform,

TABLE 4 Independent predictors for pulmonary hypertension therapy after pulmonary endarterectomy in multivariate analysis

\section{Hazard ratio $(95 \% \mathrm{CI})$}

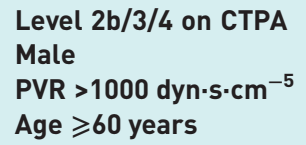

CTPA: computed tomography pulmonary angiography; PVR: pulmonary vascular resistance. p-value

$\begin{array}{lc}4.23(1.24-14.39) & 0.02 \\ 0.49(1.24-1.94) & 0.3 \\ 1.59(0.47-5.31) & 0.5 \\ 1.93(0.49-7.49) & 0.4\end{array}$


generates lower radiation and is less operator dependent. Hence, CTPA has become the standard of care for suspected acute pulmonary embolism. In our practice, CTPA has also become our standard of care for evaluation of patients with suspected CTEPH. A ventilation/perfusion scan continues to be done routinely, but we reserve conventional pulmonary angiography for cases where additional imaging confirmation is required, e.g. in the presence of a discrepancy between the ventilation/perfusion and CT scans.

The only case that was significantly misdiagnosed by imaging in this study was a patient with apparent level 1 disease on CTPA determined to be a mixing artefact when surgery revealed Jamieson type III. Although we ultimately excluded five subjects based on mixing artefacts, this case was included in the analysis because the presence of the mixing artefact was not accurately identified. This reinforces the need for optimal CT imaging in the pre-operative assessment of CTEPH. In cases where a mixing artefact is suspected, we routinely repeat the CTPA with a modified technique including increased contrast administration and delayed timing for optimal pulmonary artery opacification.

To the best of our knowledge, this is the first study that systematically correlates the surgical findings in CTEPH with the CTPA level of disease. We found that CTPA was particularly helpful to predict surgical findings in patients with segmental disease, which is important since these patients are potentially more challenging to treat surgically. Excellent outcomes can be achieved for segmental level disease at centres with high volumes and with experienced surgeons, hence emphasising the importance of high-quality imaging to identify these patients and appropriately treat them. The CTPA classification was also helpful to predict an increased risk of requiring targeted pulmonary hypertension therapy in the presence of segmental disease, which could have implications on follow-up after PEA.

This CTPA classification scheme is based on the most proximal part of the chronic thromboembolic disease and does not account for the extent of disease. In our experience, the value in defining the extent of disease based on the correlation between the pulmonary angiogram and the PVR provides limited additional information, and we pay much more attention to the location of the disease rather than its extent. Although rare, the presence of unilateral lateral disease does represent a different group of patients that should potentially be analysed separately.

Limitations in our study include the retrospective study design. However, despite this limitation, the CTPAs were reviewed by two independent radiologists blinded to the outcome of the patients. The next step would be to perform a study prospectively classifying the level of disease on CTPA using uniform criteria across multiple centres, which would potentially yield a stronger correlation with surgical findings. The CTPA findings correlated with clinical outcome and the need for targeted pulmonary hypertension therapy after PEA, suggesting that the level of disease could also have implications for the management of nonsurgical patients or for patients undergoing BPA.

In conclusion, this study demonstrates that there is strong correlation between imaging and surgical findings, particularly at a segmental level. A recognised and internationally approved radiological classification would be an important step forward in the evaluation of patients with CTEPH to recognise the disease and refine the therapeutic approaches, particularly with the advent of BPA.

Conflict of interest: M.C. McInnis reports personal fees for educational lectures from Bayer, outside the submitted work. D. Wang has nothing to disclose. L. Donahoe has nothing to disclose. J. Granton has received support through his foundation from unrestricted grants from Bayer and Jannsen Pharmaceuticals to support research, quality improvement projects and development of educational tools. He has also received payment for participation in steering and adjudication committees of clinical trials sponsored by United Therapeutics and Bellerophon. J. Thenganatt reports personal fees for advisory boards in 2019 from Janssen, outside the submitted work. K. Tan has nothing to disclose J. Kavanagh has nothing to disclose. M. de Perrot reports personal fees from Bayer, outside the submitted work.

\section{References}

1 Pengo V, Lensing AW, Prins $\mathrm{MH}$, et al. Incidence of chronic thromboembolic pulmonary hypertension after pulmonary embolism. N Engl J Med 2004; 350: 2257-2264.

2 Galiè N, Humbert M, Vachiery JL, et al. 2015 ESC/ERS Guidelines for the diagnosis and treatment of pulmonary hypertension: The Joint Task Force for the Diagnosis and Treatment of Pulmonary Hypertension of the European Society of Cardiology (ESC) and the European Respiratory Society (ERS): Endorsed by: Association for European Paediatric and Congenital Cardiology (AEPC), International Society for Heart and Lung Transplantation (ISHLT). Eur Respir J 2015; 46: 903-975.

3 Delcroix M, Lang I, Pepke-Zaba J, et al. Long-term outcome of patients with chronic thromboembolic pulmonary hypertension: results from an international prospective registry. Circulation 2016; 133: 859-871.

4 Ghofrani HA, D'Armini AM, Grimminger F, et al. Riociguat for the treatment of chronic thromboembolic pulmonary hypertension. N Engl J Med 2013; 369: 319-329.

5 Ghofrani HA, Simonneau G, D'Armini AM, et al. Macitentan for the treatment of inoperable chronic thromboembolic pulmonary hypertension (MERIT-1): results from the multicentre, phase 2, randomised, double-blind, placebo-controlled study. Lancet Respir Med 2017; 5: 785-794. 
6 Jamieson SW, Kapelanski DP. Pulmonary endarterectomy. Curr Probl Surg 2000; 37: 165-252.

7 Madani MM, Auger WR, Pretorius V, et al. Pulmonary endarterectomy: recent changes in a single institution's experience of more than 2,700 patients. Ann Thorac Surg 2012; 94: 97-103.

8 D'Armini AM, Morsolini M, Mattiucci G, et al. Pulmonary endarterectomy for distal chronic thromboembolic pulmonary hypertension. J Thorac Cardiovasc Surg 2014; 148: 1005-1011.

9 Castaner E, Gallardo X, Ballesteros E, et al. CT diagnosis of chronic pulmonary thromboembolism. Radiographics 2009; 29: 31-50

10 Thistlethwaite PA, Mo M, Madani MM, et al. Operative classification of thromboembolic disease determines outcome after pulmonary endarterectomy. J Thorac Cardiovasc Surg 2002; 124: 1203-1211.

11 Mayer E, Jenkins D, Lindner J, et al. Surgical management and outcome of patients with chronic thromboembolic pulmonary hypertension: results from an international prospective registry. J Thorac Cardiovasc Surg 2011; 141: $702-710$.

12 de Perrot $\mathrm{M}$, Thenganatt J, McRae K, et al. Pulmonary endarterectomy in severe chronic thromboembolic pulmonary hypertension. J Heart Lung Transplant 2015; 34: 369-375.

13 Ende-Verhaar YM, Meijboom LJ, Kroft LJM, et al. Usefulness of standard computed tomography pulmonary angiography performed for acute pulmonary embolism for identification of chronic thromboembolic pulmonary hypertension: results of the InShape III study. J Heart Lung Transplant 2019; 38: 731-738.

14 Rogberg AN, Gopalan D, Westerlund E, et al. Do radiologists detect chronic thromboembolic disease on computed tomography? Acta Radiol 2019; 60: 1576-1583. 